\section{THROMBOEMBOLIC EVENTS IN LEFT VENTRICULAR ENDOCARDIAL PACING: LONG-TERM OUTCOMES FROM A MULTICENTRE UK REGISTRY}

${ }^{1}$ Vinit Sawhney*, ${ }^{1}$ Guilia Domenichini, ${ }^{2}$ James Gamble, ${ }^{3}$ Guy Furniss, ${ }^{3}$ Dimitrios Panagopoulos, ${ }^{3}$ Guy Haywood, ${ }^{2}$ Kim Rajappan, ${ }^{4}$ Niall Campbell, ${ }^{1}$ Pier Lambiase, ${ }^{1}$ Simon Sporton, 'Mark Earley, 'Martin Lowe, ${ }^{1}$ Mehul Dhinoja, ${ }^{1}$ Ross Hunter, ${ }^{2}$ Tim Betts, ${ }^{1}$ Richard Schilling. ${ }^{1}$ Barts Heart Centre; ${ }^{2} J o h n$ Raddliffe Hospital; ${ }^{3}$ Plymouth Hospitals NHS; ${ }^{4}$ University Hospital South Manchester

10.1136/heartjnl-2017-311726.37

Background Endocardial left ventricular (LV) pacing is an effective alternative in patients with failed coronary sinus lead. However, the major concern is the unknown long-term thromboembolic risk and much of the data has come from a small number of centres. We examined the safety and efficacy of endocardial LV pacing across 4 UK centres, to determine the long-term thromboembolic risk.

Methods and Results Independent prospective registries from four UK centres were combied to include 68 consecutive patients from 2010-2015. Medical records were reviewed and patients were contacted for follow-up. Thromboembolic events were confirmed on imaging. Baseline patient demographics are shown in Table 1.

\section{Abstract 37 Table 1 Baseline demographics}

\begin{tabular}{|c|c|}
\hline$N=68$ & \\
\hline Age(yrs; meanะSD) & $66.8 \pm 12.3$ \\
\hline Sex(male; n, \%) & $56(82)$ \\
\hline $\begin{array}{l}\text { Cardiomyopathy(\%) } \\
\text { Ischaemic } \\
\text { Dilated }\end{array}$ & $\begin{array}{l}60 \\
40\end{array}$ \\
\hline $\begin{array}{l}\text { NYHA Grade(\%) } \\
\text { I-II } \\
\text { III } \\
\text { IV }\end{array}$ & $\begin{array}{l}10 \\
63 \\
27\end{array}$ \\
\hline $\operatorname{LVEF}(\%)$ & $27.4 \pm 7.7$ \\
\hline Intrinsic QRS duration(ms; mean $\pm S D$ ) & $159 \pm 26 \mathrm{~ms}$ \\
\hline Atrial fibrillation(\%) & 57 \\
\hline
\end{tabular}

65\% patients were already anticoagulated (39 AF, 1 DVT, 2 prosthetic valve, 2 previous LV thrombus). Mean CHADS2VASC2 score for the cohort was 3.5. 44\% patients had transventricular LV lead. The mean procedure and fluoroscopy times were $200 \pm 120$ and $32 \pm 28 \mathrm{~min}$. $75 \%$ had a siliconeinsulated pacing lead. Post-procedure, three patients had haematomas and one had tamponade requiring pericardial drain. Functional improvement was noted with decrease in mean NYHA from $3.5 \pm 1$ to $2.1 \pm 1.2 \quad(\mathrm{p}=0.001)$ and increase in LVEF from $26.5 \pm 12$ to $34 \pm 18.1(p=0.005)$ over a 20 month follow-up. Re-do procedure due to lead displacement was required in two patients. One patient underwent system extraction and surgical epicardial lead after device infection. The ischaemic stroke rate, defined as transient or permanent loss of function associated with imaging confirmation of a cerebral infarct in the appropriate territory, occurred in 4 patients $(6 \%)$ providing an annual event rate of $3.6 \%$. In multivariate analyses, the only significant correlation with the risk of CVA was sub-therapeutic INRs ( $\mathrm{p}<0.01$, CI 0.02-0.68, HR 0.12 ). There was no association between lead material and mode of delivery (transatrial/ventricular) and CVA. 14 patients died during follow-up with mean time to death post-procedure of 20 months. Cause of death was end-stage heart failure in all patients except three (pneumonia in two, knee sepsis).

Conclusion Endocardial LV lead in heart failure patients has a good success rate at 1.6 year follow-up. However, it is associated with a modest thromboembolic risk largely due to subtherapeutic anticoagulation. These results have potential for improvement and newer oral anticoagulants might play a role in this setting.

\section{A RETROSPECTIVE OBSERVATIONAL STUDY OF FLUOROSCOPY SCREENING SERVICES OF CARDIAC DEVICES IN A TERTIARY CENTRE. IS THE RADIATION WORTH IT?}

${ }^{1}$ Ayman Alatta, ${ }^{2}$ Habib Khan. ${ }^{1}$ Nevill Hall Hospital; ${ }^{2}$ Nottingham University Hospital

10.1136/heartjnl-2017-311726.38

Background All implanted cardiac devices including pacemakers and defibrillators undergo regular checks in pacemaker clinics, and often fluoroscopic screening of the devices leads is performed due to changes in pacemaker parameters such as changes in threshold, impedance and sensitivity to rule out macroscopic abnormalities with the leads.

Objective The objective of this study was to review the practice of fluoroscopic lead screening and to assess whether there is any correlation between fluoroscopic findings and device parameters abnormalities that alter patient management.

Methods We retrospectively reviewed data from reporting database and clinical letters from patients notes in 2014. 64 patients were identified that underwent fluoroscopic screening procedures during 2014. Indications for screening, screening findings and recommended actions were recorded for each procedure. Screening included 40 (53\%) complex devices, 24 (31\%) regular bradycardia devices and 12 (16\%) advisory devices including 4 Telectronics Accufix right atrial leads, and 8 St Jude Riata Right ventricular ICD leads (Table 1)

Results Fluoroscopic screening abnormalities were detected in 14 out of the 64 devices screened.There were 20 devices revision procedures performed in total. However, these were performed in only $7(50 \%)$ of those devices that showed screening abnormalities. All these devices actually showed displaced leads on screening, and loss of capture on pacemaker check, which was the main indication for screening. Continued monitoring was recommended in the other 7 devices that showed screening abnormalities. No screening abnormalities were detected in 11 devices that required revision procedures where interventions were mainly based on different electrical issues identified.Therefore, devices interventions were driven by abnormalities detected during pacemaker checks more than abnormal screening findings. 3 procedures followed screening pre box change screening which showed normal findings.

Conclusion Fluoroscopic screening of the cardiac devices is a simple procedure for imaging the device and its leads in motion. This allows for improved visualisation of the leads and identification of small abnormalities that would otherwise be missed by still radiology tests such as x-rays. In our study, there was no correlation between electrical abnormalities that initiated lead screening, and findings on fluoroscopy. Exception was in cases of loss of capture where there was almost a consistent finding of displacement of the relevant lead, albeit the number is small to make this conclusion. We concluded that screening should continue in the following settings: (1) 